\title{
EMULSIFYING PROPERTIES AND ANTIOXIDANT ACTIVITY OF PHOSPHOLIPIDS FRACTIONS FROM PALM PRESSED FIBRE
}

\author{
KGS AHMADI ${ }^{1 *}$; TETI ESTIASIH ${ }^{2}$ and AHMAD DIAUL KHULUQ ${ }^{3}$
}

\begin{abstract}
Palm pressed fibre (PPF) is a by-product of crude palm oil (CPO) extraction and has great potential as a source of phospholipids (PL). PPF was extracted from chloroform:methanol to extract total hipid, and polar lipid was separated using methanol. PL from PPF was fractionated by ethanol, and subsequently, the ethanol-soluble fraction was fractionated by acetone. Four fractions were obtained: ethanol-soluble, ethanolinsoluble, ethanol-acetone soluble, ethanol soluble-acetone insoluble. All fractions had different PL molecular species compositions. Different compositions of $P L$ in the fractions resulted in different hydrophilic-lipophilic balance (HLB) values, emulsifying properties, and antioxidant activity. HLB value of crude PL from PPF was 6.07 that was suitable for water in oil (w/o) emulsion indicated by low emulsifying activity index (EAI) and emulsion stability index (ESI) in oil-water (o/w) emulsion systems. Ethanol-soluble and ethanol-acetone soluble fractions exhibited better emulsifying properties and higher HLB values of around 10. Crude PL and its fractions showed a potential antioxidant activity to prevent bulk soybean oil in a single antioxidant system. Synergism with $\alpha$-tocopherol appeared in a binary antioxidant system indicated by low peroxide value compared to the single one. PL composition affected the ability of PL fractions as antioxidants.
\end{abstract}

Keywords: binary antioxidant system, emulsifying properties, fractionation, HLB value, palm pressed fibre.

Received: 6 July 2021; Accepted: 17 January 2022; Published online: 23 February 2022.

\section{INTRODUCTION}

Phospholipids (PL) are a mixture of phosphatecontaining lipids mainly used in the food industry as emulsifiers. Commercially, PLs are well known as lecithin, a term that is commonly used for a mixture of amphiphilic compounds that mainly

1 Department of Agroindustrial Technology,

Tribhuwana Tunggadewi University,

Jalan Tlagawarna, Tlogomas, Malang, Indonesia.

2 Department of Food Science and Technology, Universitas Brawijaya, Jalan Veteran. Malang, Indonesia.

3 Indonesian Sweetener and Fiber Crops Research Institute, Jalan Raya Karangploso, KM 4 Kotak Pos 199, Kepuh Utara, Kepuharjo, Kec. Karang Ploso, Malang, Indonesia.

* Corresponding author e-mail: kgs.ahmadi@yahoo.com comprised of PL and other minor constituents of glycolipids, carbohydrates, triglycerides, and a trace amount of water, free fatty acids (FFA), and sterols (Arnold et al., 2014). PL are also used as wetting agents, lubricants, texture modifiers, and dispersants to improve the characteristics of food products such as solubility of instant powder, less stickiness of candy, good rheology of chocolate and spreads, and others. PLs are the cell membrane constituents, and the human body recognises these compounds well, thus, PL is a safe emulsifier.

The primary source of PL is of vegetable origins, mainly oil-bearing seeds (Ahmad and $\mathrm{Xu}$, 2015) such as soybean, sunflower (Cabezas et al., 2012), canola (Xie and Dunford, 2019), rapeseed (Arnold et al., 2014), camelina seed (Belayneh et al., 2018) that are obtained during degumming in oil refining. Vegetable lecithin is a mixture of naturally occurring lipids containing more than 50\% PL 
(Robert et al., 2020). The most widely used lecithin in the food industry is soybean lecithin. PL of animal origins are the egg yolk (Bernardo et al., 2019), bovine milk (Nagai, 2012), and fish (Malaplate et al., 2019). Different sources of PL have different characteristics and emulsifying properties.

Some residual PL is also found in the industrial food wastes such as rice bran (Garba et al., 2020; Sun et al., 2020), fish discard (Topuz et al., 2021) and palm pressed fibre (PPF) (Choo et al., 2004). PPF is a by-product of the extraction process of CPO. The fibrous structure of PPF entraps 5\%$7 \%$ of the residual oil (Ooi et al., 2019) with good oxidative stability that is contributed by PL (Lau et al., 2006). PPF residual oil contains 46800 ppm of PL. The quantity of PPF PL is considerably high due to palm oil's huge world production (Choo et al., 2004). Thus, the PPF PL utilisation as the source of PL is important as an alternative to conventional PL sources.

PL are amphiphilic in nature because they have a hydrophobic tail consisting of two fatty acid chains at the sn- 1 and sn-2 positions of a glycerol moiety and a polar phosphate head group at sn-3 (Robert et al., 2020) that are attached to other hydrophilic molecules such as ethanolamine (PE), choline (PC), inositol (PI, phosphatidylinositol), serine (PS, phosphatidylserine), and glycerol (PG, phosphatidylglycerol). The most common bioactive PL in biological vegetable cells are PC, PE, PI and PS (Robert et al., 2020).

Lecithin is usually modified or fractionated to have tailor-made lecithin that is suitable for the processing and characteristics of food products. The chemical modification of PL is aimed to improve emulsifying properties by means of acid or basecatalysed hydrolysis, acetylation, hydroxylation, and hydrogenation (Li et al., 2019). Modification by enzyme is used to liberate fatty acid from sn-1 or sn-2 of the glycerol backbone of PL, resulting in better emulsifying properties for o/w emulsion (Estiasih et al., 2013). Fractionation is one way to improve the emulsifying properties of PL without modification of their chemical structures. The fractionation of PL is usually conducted by solvent to have different polarity of PL. PL are the main components of native lecithin and have different solubility in acetone or ethanol (Cabezas et al., 2012; Xie and Dunford, 2019). Solvent fractionation is used to obtain PL fractions with different compositions compared to the origin (Cabezas et al., 2012). The PPF PL is still limitedly explored, and its fractionation is important to evaluate as the alternative of commonly modified lecithin.

Lecithin fractionation aims to obtain fractions with desirable functionalities. Ethanol is commonly used as the solvent for fractionation (Xie and Dunford, 2019). PC has better solubility in alcohol than the other PL. The ethanol-soluble fraction containing a high $\mathrm{PC} / \mathrm{PE}$ ratio is found in the ethanol-soluble fraction, but the insoluble fraction has a low PC / PE ratio. PI, lysophosphatidylinositol (LPI), and phosphatidic acid (PA) are less soluble in alcohol and enriched in the ethanol-insoluble fraction (Cabezas et al., 2012). PL fractionation using ethanol and acetone as solvents is compatible with the regulations required for subsequent food and pharmaceutical applications (Bernardo et al., 2019).

The main function of PL for food, pharmaceutical, and cosmetic industries is as an emulsifier since PL naturally have amphiphilic properties. Lecithin has been widely applied in both o/w and w/o emulsions (Zembyla et al., 2020) by facilitating small droplet formation (Wu et al., 2019), positioning themselves at the interface to stabilise emulsions (Zembyla et al., 2020), and also reducing the interfacial tension (Wu et al., 2019). The advantage of lecithin over other emulsifiers is the ability to provide a strongly negative surface charge over a wide $\mathrm{pH}$ range (Warren et al., 2019). Different PL fractions exhibit different emulsifying properties. The PC-enriched fraction has improved o/w emulsifying properties; meanwhile, the fraction enriched with PI is suitable for w/o emulsions (Xie and Dunford, 2019). It is important to elucidate the suitability of PPF PL and its fractions as an emulsifier for the o/w or w/osystem.

PL has been reported to inhibit lipid oxidation in bulk oil and emulsions, but the stabilisation mechanism still remains controversial (Cardenia et al., 2011). PL are sometimes used as antioxidants in foods. Several mechanisms have been proposed for the antioxidant activity of PL. PL are chelators that could bind prooxidative metals, participating in Maillard reaction during lipid oxidation to produce antioxidative compounds, altering the other antioxidants to $\mathrm{o} / \mathrm{w}$ emulsions' interface, or in a bulk oil regenerating primary antioxidants (Cui and Decker, 2016). One mechanism of the phospholipid-tocopherol synergism is the donation of a hydrogen atom by PL to the tocopheryl radical (Doert et al., 2012). The ternary mixture of PL, tocopherol and ascorbic acid are powerful antioxidants for stabilising highly unsaturated oils (Doert et al., 2017). Antioxidant activity of PPF PL and its fractions and synergism with other antioxidants have not been evaluated yet.

The emulsifying properties and antioxidant activity of PL from PPF and its fractions are interesting to examine since PPF might be an alternative source of PL. Ethanol and acetone fractionation of PL from PPF are supposed to produce different PL molecular species compositions with different emulsifying properties and antioxidant activity. The emulsifying properties of crude PL from PPF and its fractions were evaluated in the $\mathrm{o} / \mathrm{w}$ emulsion system as a food major emulsion 
system. The antioxidant activities of crude PL and PL fractions in a single and synergistic binary system of bulk oil were also evaluated.

\section{MATERIALS AND METHODS}

\section{Materials}

PPF was kindly obtained from a palm oil milling, Surya Dumai Inc., Riau Province, Indonesia. The variety of oil palm was tenera. The PPF was stored at room temperature before being used. The chemicals for PL extraction from PPF, emulsifying properties, and antioxidant activity analysis were analytical grade from Merck (Germany). Commercial soybean oil was used for emulsifying properties and antioxidant activity analysis. The purity of acetone and ethanol for fractionation was $99.7 \%$ and $98.0 \%$, respectively. Standards for PL analysis comprised of PE (purity $\geq 98.0 \%$ by TLC), PC (purity $\geq 99.0 \%$ by TLC), PI (purity $\geq 98.0 \%$ by TLC), PA (purity $\geq 98.0 \%$ ), PG (purity $\geq 99.0 \%$ by TLC), DPG (purity $\geq 97.0 \%$ by TLC), and TLC Silica Gel G60 plates were obtained from Sigma Aldrich (Singapore).

\section{Extraction of Total Lipid from PPF}

Yunoki et al. (2008) method was used to extract total lipids from PPF (Figure 1). A total of $100 \mathrm{~g}$ of fine and dry PPF was extracted twice with $100 \mathrm{~mL}$ chloroform:methanol $(2: 1 \mathrm{v} / \mathrm{v})$, and once with 100 $\mathrm{mL}$ chloroform:methanol $(1: 2 \mathrm{v} / \mathrm{v})$, subsequently. Each extraction was conducted for $1 \mathrm{hr}$ at room temperature, and the solvent and the cake were separated by filtering through coarse filter paper. The solvent of each extract was removed with a rotary evaporator, and all lipid extracts were mixed.

\section{Separation of Crude PL from Extracted Lipid}

The Palacios and Wang (2005) method, with a slight modification, was used to separate crude PL from total lipid (Figure 2). Total lipid, $10 \mathrm{~g}$, was dissolved in $40 \mathrm{~mL}$ chloroform for $10 \mathrm{~min}$. The mixture was then centrifuged for $10 \mathrm{~min}$ at $1542 \mathrm{X} \mathrm{g}$ to separate the chloroform-soluble (supernatant) and insoluble fractions (residue or sub-natant). Chloroform from the supernatant was removed by rotary evaporation. The residue after evaporation was then extracted again with $30 \mathrm{~mL}$ of chloroform. After centrifugation at $1542 \mathrm{X}$ g for $10 \mathrm{~min}$, the chloroform from the supernatant of this second extraction was removed.

The chloroform-insoluble fraction from the first extraction was mixed with the chloroform-insoluble fraction from the second extraction. After removing the residual chloroform by rotary evaporation, the chloroform-insoluble fractions were extracted by methanol, $20 \mathrm{~mL}$, to obtain methanol-soluble fraction. Methanol was removed from this fraction, and crude PL was obtained.

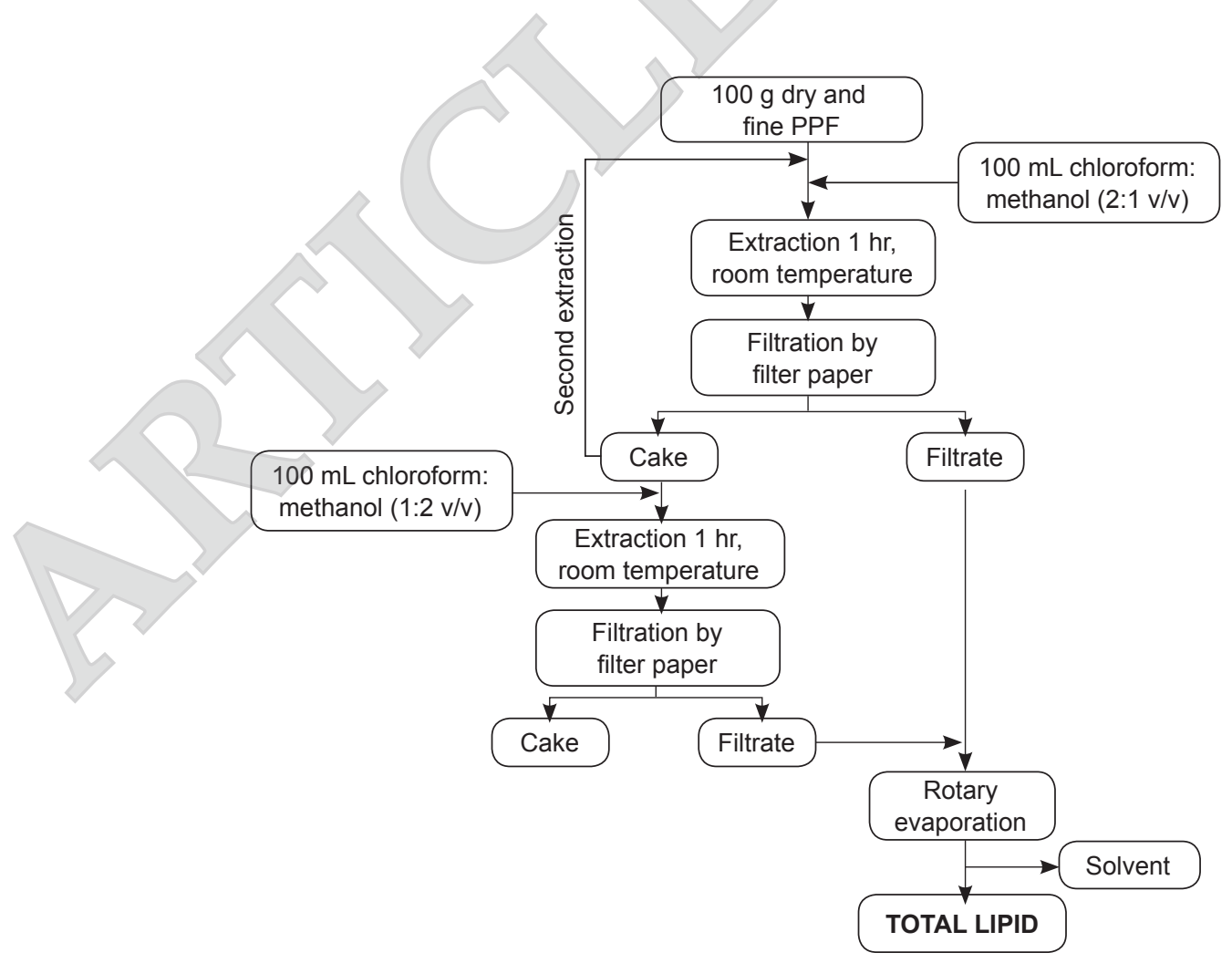

Figure 1. Total lipid extraction from PPF. 


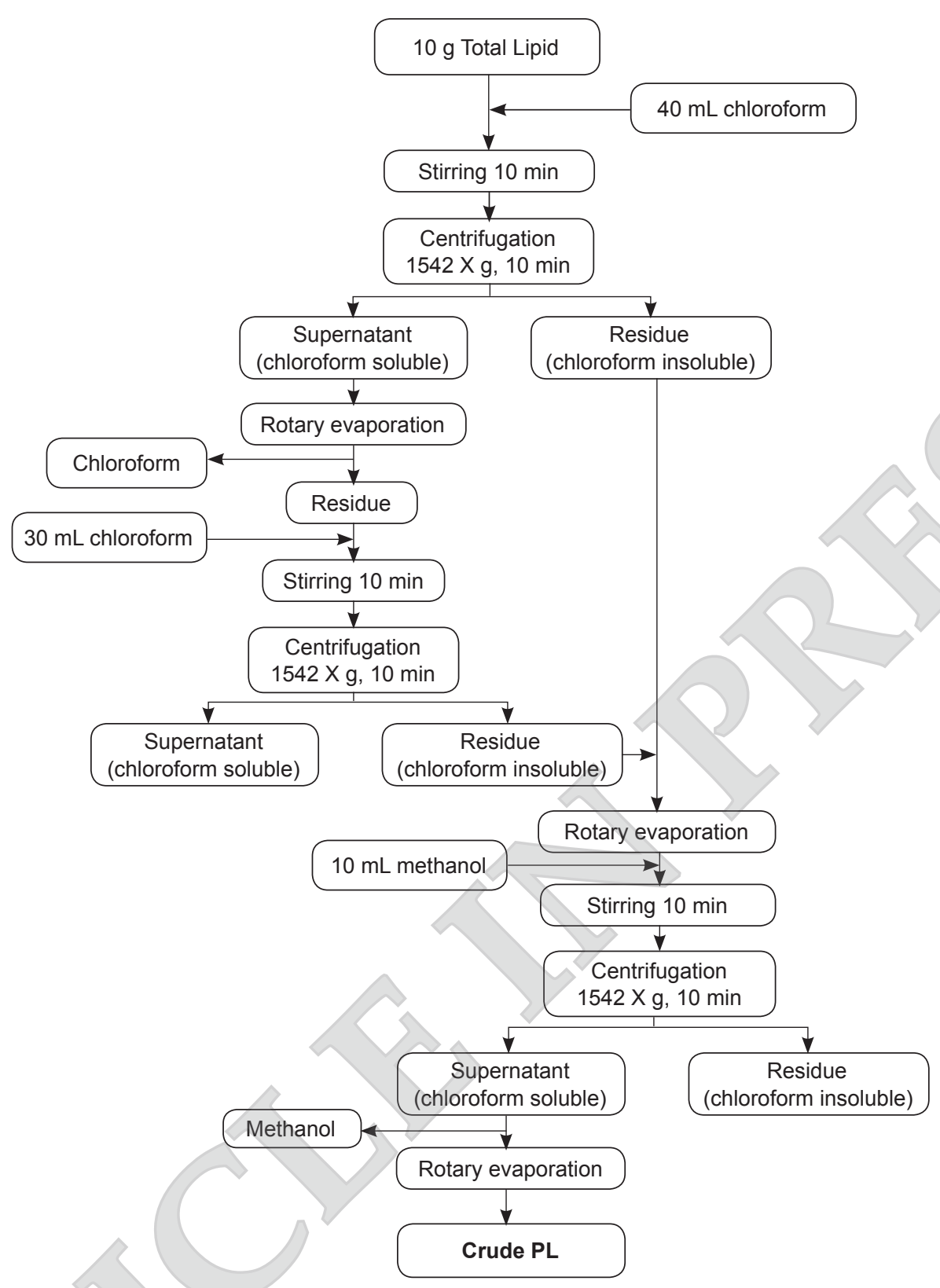

Figure 2. Separation of crude PL from the total lipid of PPF.

\section{PL Fractionation}

The fractionation of PL was conducted by using acetone and ethanol at room temperature (Figure 3). Extraction by ethanol was used as the first fractionation. Crude PL, $5 \mathrm{~g}$, was dissolved in $20 \mathrm{~mL}$ ethanol and agitated for $60 \mathrm{~min}$ by using a magnetic stirrer. The mixture was centrifuged for $10 \mathrm{~min}$ at $15000 \mathrm{X} \mathrm{g}$. The residue was an ethanolinsoluble fraction, and the supernatant contained an ethanol-soluble fraction. A rotary evaporator removed ethanol from the supernatant and residual ethanol in an ethanol-insoluble fraction to obtain ethanol-soluble and ethanol-insoluble fractions.

Some of the ethanol-soluble fraction was taken and further fractionated by using acetone through dissolving in acetone at a ratio of the ethanol-soluble fraction to acetone 1:4 (w:v). The mixture was agitated for $60 \mathrm{~min}$ by a magnetic stirrer. Acetone soluble fraction and acetone-insoluble fraction were separated by centrifugation at $15000 \mathrm{Xg}$ for $10 \mathrm{~min}$. A rotary evaporator removed the residual acetone from each fraction. By solvent fractionation, four fractions were obtained: ethanol-soluble fraction, ethanol-insoluble fraction, ethanol-acetone soluble fraction, and ethanol soluble-acetone insoluble fraction; and crude PL without fractionation as a control.

\section{Analysis of PL Profile}

The analysis of the PL profile was according to the method of Nzai and Proctor (1998). This analysis used silica gel G60 plates with a 


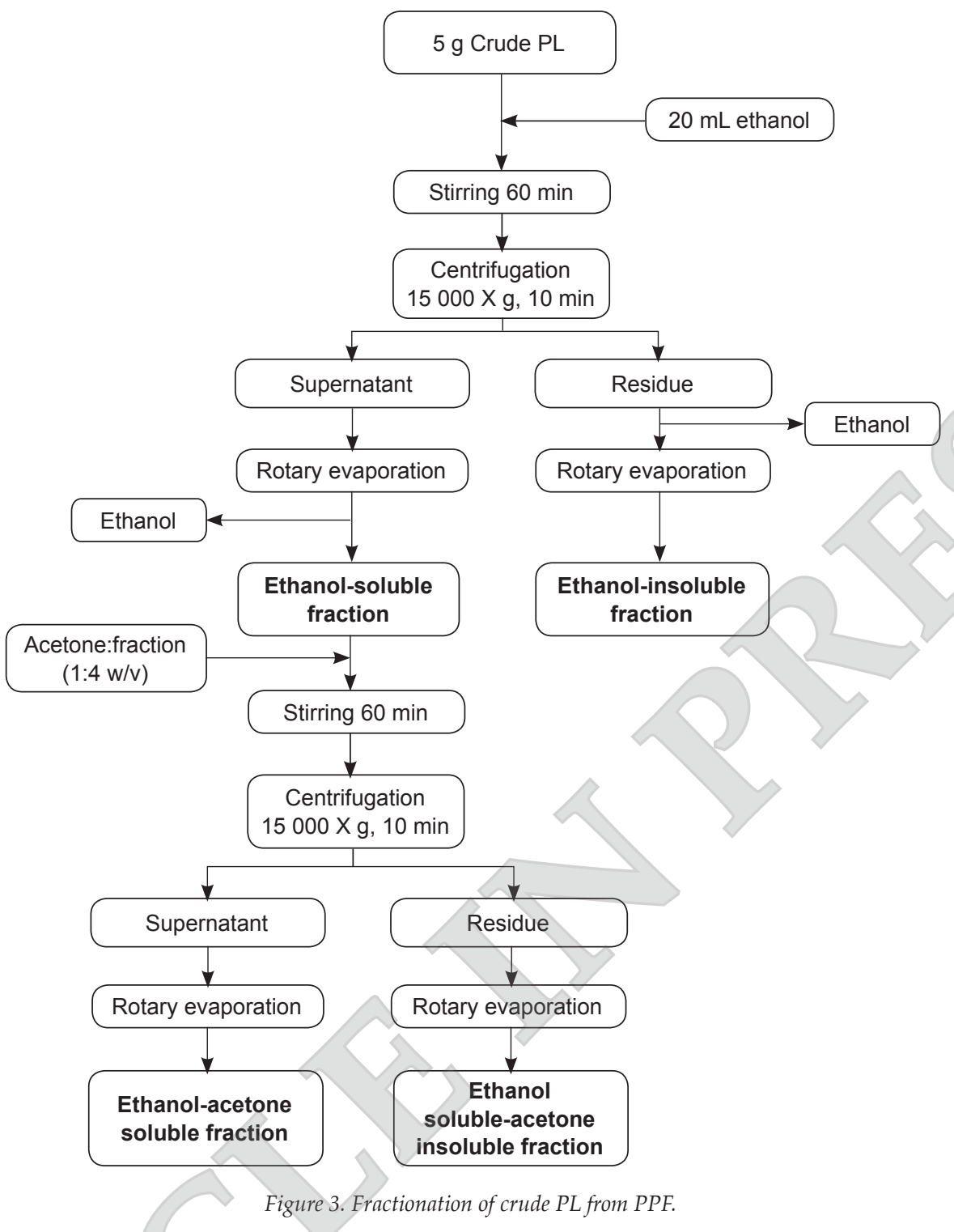

chloroform:methanol:water (75:25:3 v/v/v) system as a developing solution. Each standard PL, crude $\mathrm{PL}$, and the fractions were dissolved separately in a chloroform:methanol solution $(95: 5 \mathrm{v} / \mathrm{v})$ at $1 \mathrm{mg} \mathrm{mL}^{-1}$ concentration. After activating the plates by drying for $20 \mathrm{~min}$ in an oven at $100^{\circ} \mathrm{C}$, the PL standard, crude $\mathrm{PL}$, and the fraction solution in a volume of $10 \mu \mathrm{L}$ were spotted separately. The plate development was run for $40 \mathrm{~min}$, and then the plates were dried at room temperature for $10 \mathrm{~min}$, and heated in the oven for $10 \mathrm{~min}$ at $90^{\circ} \mathrm{C}$. TLC scanner (CAMAG TLC Scanner 3) was used for the quantification of the molecular species of PL. PL concentration in the samples was calculated on the basis of area curves of each PL from the densitogram of the TLC scanner.

\section{Analysis of Emulsifying Properties}

Hydrophilic lipophilic balance (HLB). The suitability of PL from PPF and its fractions to stabilise emulsions are measured by HLB value. HLB plays an essential role in balancing the interfacial tension between oil and water in an emulsion. The HLB value permits the ability of an emulsifier to stabilise w/o or o/w emulsions (Alam et al., 2020). HLB value ranges from 0 to 20 and was measured based on saponification number $(S)$ (AOAC, 1997) and acid value $(A v)($ AOAC, 1980) with the Equation (1) as follows:

$$
\text { HLB }=20\left(1-\frac{S}{A v}\right)
$$

Emulsifying activity index (EAI) and emulsifying stability index (ESI). In this study, the capability of crude PL and its fraction in stabilising $\mathrm{o} / \mathrm{w}$ emulsions was evaluated by EAI and ESI. The EAI represents the emulsifiers' ability to be absorbed in the interface during the emulsion formation, and a higher EAI represents a stronger emulsification capability (Chen et al., 2020). The emulsifying 
activity index represents the surface area covered by the emulsifier. Thus, the higher EAI value indicates the emulsifier's greater ability to produce smaller droplets of the dispersed phase. The turbidity method was used to measure EAI and ESI of crude PL from PPF and its fractions (Pearce and Kinsella, 1978). The emulsion was prepared by mixing $0.5 \mathrm{~g}$ of soybean oil with $9.5 \mathrm{~mL}$ of distilled water to have an oil fraction of $0.5 \%$. As much as $0.05 \mathrm{~g}$ crude PL or its fractions was added to the mixture of oil and water. The mixture was homogenised by agitating with a homogeniser (ultra turrax) at a speed of $12500 \mathrm{rpm}$ for $1 \mathrm{~min}$. EAI and ESI were measured by mixing $0.12 \mathrm{~mL}$ of emulsion with phosphate buffer $(0.005 \mathrm{M}, \mathrm{pH} 7)$ containing sodium dodecyl sulphate (SDS) $0.3 \%$ to a volume of $10 \mathrm{~mL}$, to have an oil volume fraction of $6 \times 10^{-5}$. When emulsion and phosphate buffer were mixed, the time was $t_{0}$ and the absorbance at $500 \mathrm{~nm}$ was $\mathrm{A}_{0}$. The mixture was allowed to stand for $10 \mathrm{~min}\left(\mathrm{t}_{10}\right)$, and the absorbance at $t_{10}$ was expressed as $A_{t}$. The following Equation (2) was used to calculate EAI $\left(\mathrm{m}^{2} \mathrm{~g}^{-1}\right)$ and ESI (min):

$$
\mathrm{EAI}=\frac{(2.303)(2)\left(\mathrm{A}_{0} \mathrm{X} \text { dilution factor }\right)}{\mathrm{CX} \Phi \mathrm{X} 10000}
$$

$\mathrm{A}_{0}=$ absorbance at $\min 0$

$\mathrm{C}=$ emulsifier concentration $(\mathrm{g} / \mathrm{mL})$

$\Phi=$ oil volume fraction

$\mathrm{A}_{\mathrm{t}}=$ absorbance at $\min 10$

$\Delta \mathrm{t}=10 \mathrm{~min}$

$$
\mathrm{ESI}=\mathrm{A}_{0} \mathrm{X} \frac{\Delta \mathrm{t}}{\mathrm{A}_{0}-\mathrm{A}_{\mathrm{t}}}
$$

\section{Antioxidant Activity}

The antioxidant activity measurement was based on the ability to inhibit lipid peroxidation of bulk soybean oil. The ability of crude PL and its fraction as a single antioxidant was measured in $5 \mathrm{~mL}$ of soybean oil that was added by crude PL or its fractions at concentration of $0.5 \%$ or $5000 \mathrm{ppm}(\mathrm{w} / \mathrm{v})$. The mixture was incubated at $37^{\circ} \mathrm{C}$ for five days, and every day a sample was removed to measure the peroxidation value by the ferric thiocyanate method (Hills and Thiel, 1946). For binary antioxidant systems, crude PL and its fraction were added separately to soybean oil. $\alpha$-tocopherol of 1000 ppm was added to the system and homogenised by agitation with a homogeniser (ultra turrax) at the speed of $1500 \mathrm{rpm}$. The mixture was incubated for five days, and every day, the peroxide value was measured. Commercial antioxidant BHT at a concentration of $100 \mathrm{ppm}$ and $\alpha$-tocopherol at a 1000 ppm concentration were used as controls.

\section{Statistical Analysis}

Correlations between PL molecular species from PPF PL fractions and EAI, ESI, and the mean of peroxide values during five days incubation in single and antioxidant systems were conducted by multivariate Pearson Correlation using IBM SPSS Statistic 24. All the treatments were replicated three times. The data were analysed by one-way analysis of variance using SPSS software, and Duncan Multiple Range Test further analysed the differences among fractions at a significance level of $5 \%$.

\section{RESULTS AND DISCUSSION}

\section{PL Composition of Crude PL from PPF and its Fractions}

Crude PL and its fractions had a different PL profile (Table 1). Predominant PL in the crude PL from PPF were PC, followed by PG, PE, DPG, PI and PA in decreasing order of concentration. Three species of PL and their concentrations from PPF extracted by hexane were $\mathrm{PC}>\mathrm{PE}>\mathrm{PG}$, and four species of $\mathrm{PL}$ were found in the ethanol extract $(\mathrm{PC}>\mathrm{PE}>\mathrm{PG}>\mathrm{PA}$ ) (Choo et al., 2004). Meanwhile, Choo et al. (2004) reported four species of PL in PPF, which were PG $>$ PC $>$ PE $>$ LPC (lysophosphatidylcholine). The concentration of PL in the ethanol extract was much higher than in hexane extract. This study found more molecular species of PL. Gradual extraction using chloroform:methanol to extract total lipids and separation of polar lipids using methanol from chloroform-insoluble fraction resulted in more PL species. The purity of PL extract from PPF was $61.67 \%$. Choo et al. (2004) reported that the concentration of PL in the ethanol extract was $4.68 \%$ and in the hexane extract was $0.14 \%$. Chua et al. (2009) extracted the PL from PPF by chloroform:methanol 2:1 (v/v), and the concentration of PL in the extract ranged from $1.01 \%$ to $5.33 \%$, depending on the ratio of dry PPF to solvent $(\mathrm{w} / \mathrm{v})$.

The FFA found in the extract were impurities. FFA were more polar than triglycerides that were soluble in the methanol during PL separation. Less polar PL, such as PG, DPG, PI and PA, were found in the extract. The extraction of total lipid from PPF used the mixture of chloroform and methanol. Polar and nonpolar lipids were extracted in this step. Separation of PL from total lipids was conducted by chloroform that PL was insoluble and more polar residue than chloroform soluble matters. Further extraction by methanol extracted more polar lipid such as PC. However, less polar PL including PE, PG, DPG, PI and PA were also found due to the suitable polarity with methanol. 
Fractionation by ethanol resulted in an increased purity of the PL (Table 1). PC increased dramatically in the ethanol extract compared to the original crude PL. Lower PI, PE, PG and DPG were found meanwhile, PA concentration was similar to the origin. The concentration of PC increased in the ethanol-soluble fraction. On the other hand, the ethanol-insoluble fraction was enriched with PI and PE (Cabezas et al., 2009). In this study, the purity of the ethanol-insoluble fraction was higher than that of the soluble one. A sharp increase of PE and PI was found in the ethanol-insoluble fraction. This finding is in accordance with the results of the study by Xie and Dunford (2019) that PE, PI and LPI increased in ethanol-insoluble fraction. This finding was in accordance with the Palacios and Wang (2005) report that PC increased after ethanol extraction. In this study, the PL quantification was based on the percentage of each PL peak area curve. The decrease in one species of PL would increase other species of PL. PE, PG and DPG concentration increased in ethanol insoluble fraction, indicating that the ethanol-insoluble fraction contained higher, less polar PL.

Further fractionation of ethanol-soluble fraction by acetone resulted in an acetone soluble fraction that was enriched with PC. Meanwhile, the ethanol soluble-acetone insoluble fraction was rich in PI, PE, PG and DPG. The concentration of more polar PL was found in the ethanolacetone soluble. In contrast, the concentration of less polar PL was found in the ethanol solubleacetone insoluble fraction. Acetone and ethanol have different polarities in that ethanol is more polar than acetone. Acetone is usually used to purify PL so that the acetone insoluble fraction matters indicate the PL's purity. Acetone is used to separate nonpolar lipids from the PL. Therefore, the less polar PL species were found in the acetoneinsoluble fraction.
Further fractionation of ethanol-soluble fraction by acetone resulted in an acetone soluble fraction that was enriched with PC. Meanwhile, the ethanol soluble-acetone insoluble fraction was rich in PI, PE, PG and DPG. The concentration of more polar PL was found in the ethanol-acetone soluble. In contrast, the concentration of less polar PL was found in the ethanol soluble-acetone insoluble fraction. Acetone and ethanol have different polarities in that ethanol is more polar than acetone. Acetone is usually used to purify PL so that the acetone insoluble fraction matters indicate the PL's purity. Acetone is used to separate nonpolar lipids from the PL. Therefore, the less polar PL species were found in the acetone-insoluble fraction.

\section{Emulsifying Properties of Crude PL from PPL and its Fractions}

An emulsion contains two immiscible liquids that are thermodynamically unstable. One way to stabilise emulsions is to reduce interfacial tension in the boundary between the immiscible liquids by using emulsifiers. An emulsifier's effectiveness to stabilise emulsions might be affected by several factors, such as HLB value, concentration, and emulsifier flexibility to reposition at the interface (Feng et al., 2018). The HLB values of crude PL from PPF and its fraction are shown in Table 2.

The crude PL from PPF had a low HLB value of 6.07 that might be contributed by PL molecular species composition (Table 1). The predominant PL in crude PL from PPF were less polar PL, such as PA, PG, DPG, PI and PE, which caused hydrophobic moieties to dominate, resulting in low HLB value. Some lecithin ingredients are not particularly good at stabilising $\mathrm{o} / \mathrm{w}$ emulsions when used in isolation because of low or intermediate HLB numbers of 2 to 8 (McClements and Gumus, 2016). Thus, the crude PL from PPF might be suitable for w/o emulsion.

TABLE 1. PL PROFILE (\%) CRUDE PL FROM PPF AND ITS FRACTIONS

\begin{tabular}{|c|c|c|c|c|c|}
\hline \multirow[b]{2}{*}{ PL } & \multirow[b]{2}{*}{$\begin{array}{c}\text { Crude PL } \\
(\%)\end{array}$} & \multicolumn{4}{|c|}{ Fractions $(\%)$} \\
\hline & & Ethanol soluble & $\begin{array}{c}\text { Ethanol } \\
\text { insoluble }\end{array}$ & $\begin{array}{c}\text { Ethanol-acetone } \\
\text { soluble }\end{array}$ & $\begin{array}{c}\text { Ethanol } \\
\text { soluble-acetone } \\
\text { insoluble }\end{array}$ \\
\hline Free fatty acid (FFA) & $18.61 \pm 0.87 c$ & $21.28 \pm 0.98 b$ & $10.53 \pm 0.46 \mathrm{~d}$ & $24.21 \pm 1.04 a$ & $11.19 \pm 0.54 \mathrm{~d}$ \\
\hline Phosphatydic acid (PA) & $7.02 \pm 0.51 c$ & $7.44 \pm 0.24 b$ & $7.36 \pm 0.36 b c$ & $7.65 \pm 0.34 \mathrm{ab}$ & $8.34 \pm 0.56 a$ \\
\hline Phosphatidylglicerol (PG) & $21.24 \pm 1.65 b$ & $15.79 \pm 0.98 c$ & $24.67 \pm 1.23 a$ & $14.46 \pm 0.88 c$ & $21.72 \pm 1.02 b$ \\
\hline Diphosphatidylglicerol (DPG) & $8.88 \pm 0.26 c$ & $7.92 \pm 0.56 \mathrm{~d}$ & $17.38 \pm 0.32 b$ & $7.72 \pm 0.44 \mathrm{~d}$ & $18.18 \pm 0.56 a$ \\
\hline Phosphatidylethanolamine (PE) & $13.21 \pm 1.01 \mathrm{~b}$ & $3.31 \pm 0.23 \mathrm{~d}$ & $13.13 \pm 0.89 b$ & $7.47 \pm 0.78 c$ & $16.31 \pm 1.24 \mathrm{a}$ \\
\hline Phohatidylinositol (PI) & $8.87 \pm 0.47 c$ & $3.23 \pm 0.28 \mathrm{e}$ & $21.28 \pm 0.89 a$ & $3.91 \pm 0.13 d$ & $16.72 \pm 1.12 b$ \\
\hline Phosphatidylcholine (PC) & $22.17 \pm 1.09 c$ & $41.03 \pm 2.34 \mathrm{a}$ & $3.65 \pm 0.23 e$ & $34.58 \pm 1.78 b$ & $7.54 \pm 0.65 d$ \\
\hline Purity (\%) & $61.67 \pm 1.05$ & $65.78 \pm 0.78$ & $71.44 \pm 0.67$ & $67.06 \pm 0.59$ & $68.49 \pm 0.98$ \\
\hline
\end{tabular}

Note: The data with different notification means different significantly at $\alpha-<0.05$ for the same row. 
TABLE 2. EMULSIFYING PROPERTIES AND ANTIOXIDANT ACTIVITY OF CRUDE PL FROM PPF AND ITS FRACTIONS

\begin{tabular}{|c|c|c|c|c|c|}
\hline \multirow{2}{*}{ PL and fraction } & \multirow{2}{*}{ HLB value } & \multirow{2}{*}{$\operatorname{EAI}\left(\mathrm{m}^{2} \mathrm{~g}^{-1}\right)$} & \multirow{2}{*}{ ESI (min) } & \multicolumn{2}{|c|}{$\begin{array}{l}\text { Average PV (meq } \mathrm{kg}^{-1} \text { ) during } \\
\text { 5-day storage }\end{array}$} \\
\hline & & & & $\begin{array}{l}\text { Single antioxidant } \\
\text { system }\end{array}$ & $\begin{array}{c}\text { Binary antioxidant } \\
\text { system }\end{array}$ \\
\hline Crude PL & $6.07 \pm 0.24 c$ & $197.80 \pm 7.89 c$ & $62.51 \pm 1.29 c$ & $5.20 \pm 0.23 c$ & $4.18 \pm 0.11 c d$ \\
\hline Ethanol soluble & $10.85 \pm 0.58 \mathrm{a}$ & $282.36 \pm 2.98 b$ & $78.43 \pm 2.21 b$ & $6.70 \pm 0.37 \mathrm{a}$ & $5.12 \pm 0.23 b$ \\
\hline Ethanol insoluble & $5.68 \pm 0.16 b$ & $196.88 \pm 3.45 c$ & $52.70 \pm 1.87 \mathrm{~d}$ & $5.35 \pm 0.52 b c$ & $4.33 \pm 0.16 c$ \\
\hline Ethanol-acetone soluble & $10.11 \pm 0.78 \mathrm{a}$ & $309.81 \pm 9.88 \mathrm{a}$ & $94.07 \pm 3.43 a$ & $5.80 \pm 0.26 b$ & $4.27 \pm 0.11 c$ \\
\hline Ethanol soluble-acetone insoluble & $5.54 \pm 0.22 b$ & $179.49 \pm 4.56 \mathrm{~d}$ & $43.89 \pm 2.01 \mathrm{e}$ & $5.87 \pm 0.32 b$ & $4.05 \pm 0.11 d$ \\
\hline$\alpha$-tocopherol & & & & $6.30 \pm 0.66 a b$ & $6.55 \pm 0.22 a$ \\
\hline Butylated hydroxytoluene & & & & $3.63 \pm 0.40 \mathrm{~d}$ & $3.83 \pm 0.21 \mathrm{e}$ \\
\hline
\end{tabular}

Note: The data with different notification means different significantly at $\alpha-<0.05$ for the same column.

The fractionation by ethanol increased the HLB value of the ethanol-soluble fraction to 10.85 , which is suitable for the $\mathrm{o} / \mathrm{w}$ emulsion (Alam et al., 2020). Increasing HLB value was related to the dramatic increase in PC, meanwhile less polar PL decreased. Analysis of Pearson correlation showed that PC positively correlated with HLB value. The ethanol-insoluble fraction had a significantly lower HLB value of $5.68(\alpha<0.05)$ than the HLB value of the original crude PL from PPF. A sharp increase in concentration was found in PI and DPG.

Meanwhile, other less polar PL increased to some extent. Increasing PI and DPG might lower HLB values, as indicated by a negative Pearson correlation between both with HLB value. The HLB value of the ethanol soluble-acetone insoluble fraction was not significantly different from that of the ethanolinsoluble fraction. The composition of PL in both fractions was similar, which was lacking in PC but rich in the less polar PL. Meanwhile, the ethanolacetone soluble fraction had an increased HLB value compared to the crude PL from PPF. This HLB value was not significantly different $(\alpha<0.05)$ from that of the ethanol-soluble fraction. Both fractions were rich in $\mathrm{PC}$, and the composition of $\mathrm{PL}$ in the fractions was also almost similar. Based on the HLB value, fractionation of crude PL from PPF by ethanol and acetone produced fractions with different HLB values. However, the ethanol-soluble and ethanolacetone soluble HLB values were not significantly different, and the same characteristics were found for ethanol insoluble and ethanol soluble-acetone insoluble fractions. Therefore, further acetone fractionation after ethanol fractionation was not required because no significant HLB values were produced.

Different fractions of PL from PPF showed different PL compositions that might result in different emulsifying properties. Data in Table 2 show that PL fractions which were able to create the highest specific surface area were found in the ethanol-acetone soluble fraction. EAI of this fraction was significantly higher than that of the ethanolsoluble fraction, although the HLB values of both fractions were similar. Although the ethanol-acetone soluble fraction had a lower PC concentration (Table 1), it had a higher purity and lower concentrations of less polar PL such as DPG and PG, thus, revealing better emulsifying properties than the ethanol-soluble fraction. PL with a high PC level was reported to produce smaller oil droplets (Cabezas et al., 2012). Analysis of Pearson correlation showed that PC attributed positively with EAI. Other PL had a negative correlation. Meanwhile, the presence of a high amount of PI corresponded to w/o emulsions (Belayneh et al., 2018), which means the lower concentration of PI in ethanol-acetone soluble fraction resulted in better emulsifying properties for $\mathrm{o} / \mathrm{w}$ emulsion.

The EAI of the ethanol-insoluble fraction was not significantly different from that of crude PL from PPF, although both PL compositions were also different. Crude PL had higher PC than the ethanolinsoluble fraction, but the levels of PI and PG, as less polar PL, were much lower. Thus, the EAI values of both were similar. PI, PG, DPG and PA are less polar PL suitable for w/o emulsions; therefore, the capability to create smaller droplets in o/w emulsion was lower. The ethanol soluble-acetone insoluble fraction showed the lowest EAI and also had the lowest HLB value. This fraction was suitable for w/o emulsion because PG and other less polar PL dominated the PL composition.

The ESI indicates the emulsifier capability to remain at the $(\mathrm{o} / \mathrm{w})$ interface in emulsions after a period of storage(Chen et al.,2020). Emulsion stability is related to PL's molecular species composition (McClements and Gumus, 2016). The molecular composition of the PL affects emulsion stability (Guiotto et al., 2013). Data in Table 2 show that the ESI of crude PL and its fraction was in accordance with the EAI. The highest EAI of PL fraction also 
revealed the highest ESI. The smaller droplets produced during emulsification that was stabilised by PL fractions resulted in higher emulsion stability. The highest ESI was found in the ethanol-acetone soluble fraction, and the order pattern of ESI of each PL fraction was similar to the EAI. The PL molecular species composition similarly affected ESI and EAI. PL fractions containing a high level of PC produced $\mathrm{o} / \mathrm{w}$ emulsions with high stability, and PL fractions containing less polar PL resulted in lower o/w emulsion stability. Analysis of Pearson correlation showed that PC had a positive contribution to ESI. Meanwhile, other PL showed a negative correlation.

\section{Antioxidant Activity}

Some studies revealed that PL has the ability to prevent oxidation in $\mathrm{o} / \mathrm{w}$ emulsion (Cardenia et al., 2011) and bulk oil (Cui et al., 2014; Charanyaa et al., 2019). In this study, crude PL capability from PPF and its fractions to inhibit oxidation was evaluated in bulk soybean oil. The primary oxidation products (peroxide value, pv) were monitored during 5 daystorage at $37^{\circ} \mathrm{C}$. A commercial synthetic antioxidant butylated hydroxytoluene (BHT) and a natural antioxidant $\alpha$-tocopherol were used as controls.

In a single antioxidant system, crude PL and its fractions revealed antioxidant activity (Figure 4). Their activity at a concentration of 5000 ppm was comparable to BHT's antioxidant activity at 100 ppm. $\alpha$-tocopherol at a $1000 \mathrm{ppm}$ concentration failed to retard oxidation indicated by a sharp increase of peroxide value after 4-day storage. The ethanol-insoluble fraction showed the highest antioxidant activity among PL fractions and always had the lowest daily peroxide value. Except for the ethanol-insoluble fraction, crude PL from PPF and its fractions did not show significantly different antioxidant activity. Fractionation by ethanol and acetone did not produce fractions with better inhibition of peroxidation.

The groups of PE and PA in PL structures significantly contributed to the antioxidant activity, while inositol showed little effect (Doert et al., 2017). The PL fraction with the highest PI (ethanol-insoluble fraction) showed the lowest antioxidant activity in this study. Cardenia et al. (2011) reported that PL's antioxidant activity was affected by the type of phosphate head group. PC with oleic or palmitic acids are the most effective antioxidant to inhibit hydroperoxide formation in an emulsion. The PL antioxidant activity is related to their ability to form a layer at the emulsion droplets interface or to chelate metals. A physical barrier to oxygen contributes to PC's antioxidant activity in the w/oemulsion (Choe and Choe, 2016). The different structures of emulsions and bulk oil might result in different mechanisms of PL inhibition to oxidation. In this study, PC and PA showed a positive correlation with average daily peroxide values; other PL were negatively correlated.

Some mechanisms have been proposed for the antioxidant activity of PL. Some PL effectively inhibits lipid oxidation in emulsion due to their capacity to scavenge free radicals (McClements and Gumus, 2016) by hydrogen donation (Choe and Choe, 2016). PL could chelate prooxidative metals, alter other antioxidant locations, regenerate primary antioxidants, and produce antioxidative compounds through Maillard reactions during lipid oxidation (Cui and Decker, 2016). In this study, the proposed mechanism of antioxidant activity of crude PL from PPF and its fractions was scavenging activity because no prooxidant metal and primary antioxidants were added to the bulk oil, and there was no protein or amine as precursors for Maillard reaction in the system.

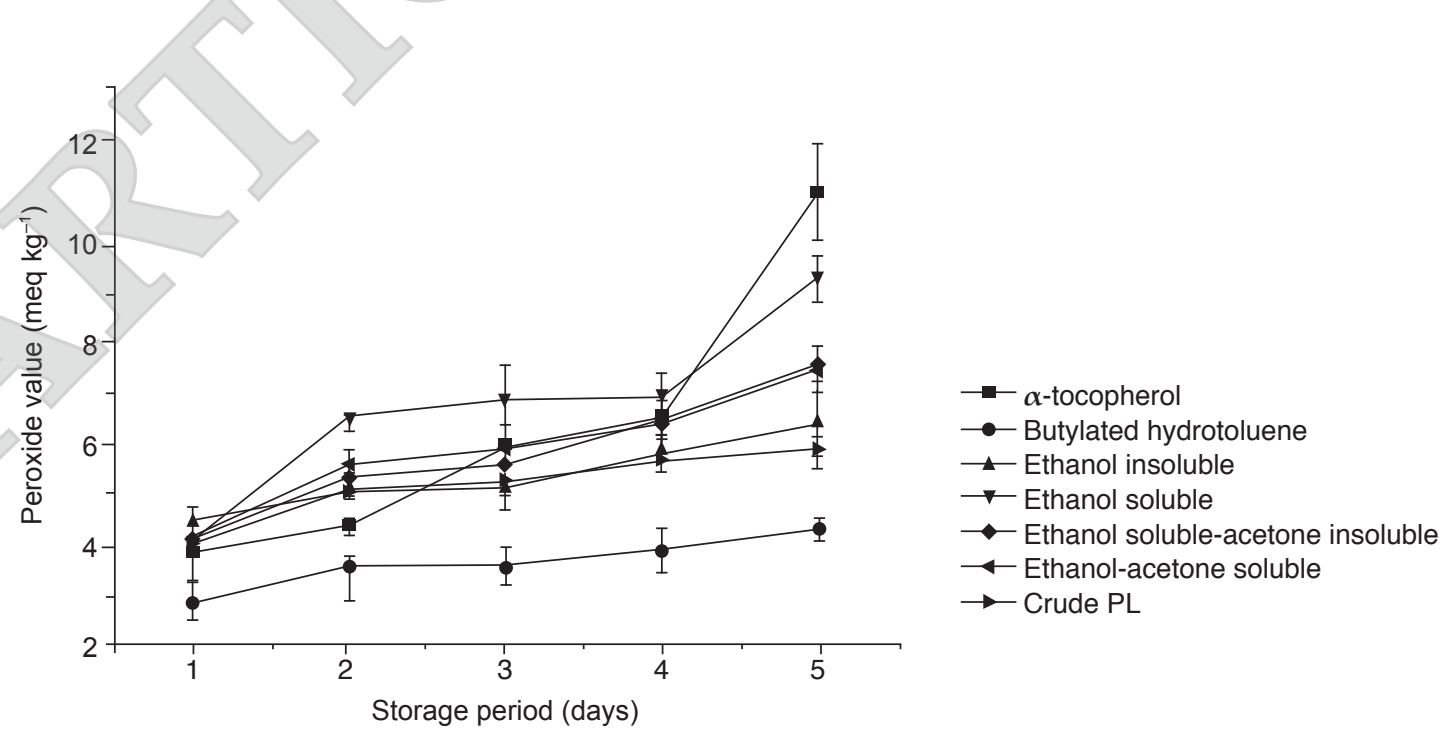

Figure 4. The effect of crude PL from PPF and its fractions in inhibiting peroxidation of bulk soybean oil during 5-day storage in a single antioxidant system. The concentration of crude PL and its fractions was 5000 ppm, BHT was 100 ppm, and $\alpha$-tocopherol was 1000 ppm. 
Crude PL and its fractions were added to soybean oil to evaluate their ability to regenerate the primary antioxidant $\alpha$-tocopherol. Figure 5 shows that $\alpha$-tocopherol in the bulk oil-PL system produced lipid oxidation of $4.30 \%$ to $35.27 \%$ lower than lipid oxidation of a single antioxidant system, depending on the PL fractions. All of the PL fractions and crude PL exhibited synergism with $\alpha$-tocopherol, which was indicated by lower daily peroxide values of soybean oil added by the binary antioxidant system. The reductions were $4.30 \%$ for insoluble ethanol fraction, $35.27 \%$ for ethanol-soluble fraction, $26.32 \%$ for ethanol-acetone soluble fraction, $30.96 \%$ for ethanol soluble-acetone insoluble fraction, and $19.52 \%$ for crude PL. The lowest antioxidant activity was found in the binary system stabilised by the ethanol-soluble fraction, and the highest one was produced by the ethanol soluble-acetone insoluble fraction (Table 2). Other PL fractions did not show a significant difference in antioxidant activity.

PL molecular species composition affected the antioxidant activity in the binary system. The ethanol soluble-acetone insoluble fraction had the highest level of PE. PE is a PL species with an amine group that has the ability to donate $\mathrm{H}^{+}$to a tocopheryl radical. PL and phenolic antioxidants such as $\alpha$-tocopherol synergistically inhibit lipid oxidation (Doert et al., 2012). PE and PS can regenerate $\alpha$-tocopheryl quinone radical to $\alpha$-tocopherol. As a non-amine PL, PC does not show a synergistic effect on lipid oxidation inhibition (Doert et al., 2017). The ethanol soluble-acetone insoluble fraction had the highest level of PE and showed the highest antioxidant activity. Meanwhile, the ethanol-soluble fraction had the highest PC level and exhibited the lowest antioxidant activity in the binary system compared to other fractions.
All of the PL fractions had similar levels of PA, and presumably, this PL had an equal contribution to the antioxidant activity of each fraction. Phosphoric moiety in PA contributes to the antioxidant activity of PL (Doert et al., 2017). PA acts as an antioxidant synergist and acidity regulator (Silva and Lidon, 2016). PA from PL fractions might also regenerate $\alpha$-tocopherol, thus having a role in lowering the peroxidation of soybean oil with a binary antioxidant system. Other PL species, PG and DPG, had not been reported for their role as antioxidants. Both had glycerol moiety, which might have no role in regenerating primary antioxidants, such as inositol in PI. PI has been reported to have no capability in regenerating primary antioxidants (Doert et al., 2012).

\section{Process for Scaling Up}

Before scaling up, it is very important to consider the feasibility of PL production from PPF and its fractionation. Production of PL from PPL requires some equipment in the pilot plant scale, including a mixing tank to extract the total lipid, separation of PL from total lipid, and fractionation. A pilotscale centrifuge is required to separate supernatant and sub-natant (residue) in total lipid extraction, PL separation, and fractionation. Evaporation solvent or residual solvent from total lipid, crude PL, and its fractions after centrifugation required a pilot-scale rotary evaporator. Scale-up is very important for commercial production of such products, including PL and its fractions from PPF. Some parameters should be established in scaling up, such as time for extraction, separation, centrifugation, and the speed for stirring and centrifuging. Therefore, further study is required to produce PL from PPF and its fractions on a pilot plant and commercial scale.

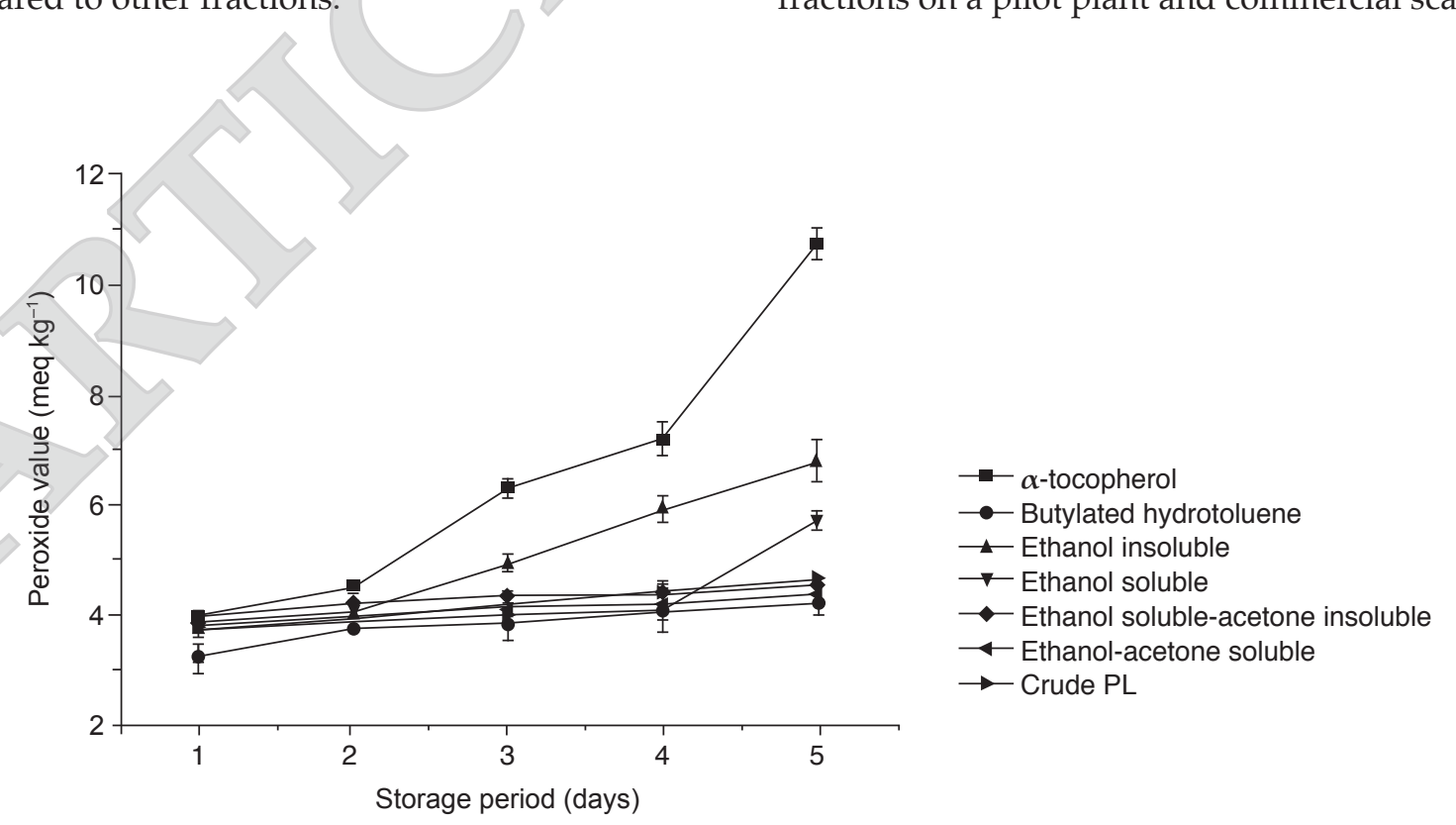

Figure 5. The effect of crude PL from PPF and its fractions (each was $5000 \mathrm{ppm}$ ) in inhibiting peroxidation of bulk soybean oil during 5-day storage in binary antioxidant systems with $\alpha$-tocopherol of $1000 \mathrm{ppm}$. BHT of $100 \mathrm{ppm}$ was used as control. 


\section{CONCLUSION}

PPF could be used as the source of PL. Crude PL from PPF and its fractions had different PL profiles, and the predominant PL in crude was PG, followed by PC, PE, DPG, PI and PA. Fractionation by ethanol resulted in the increasing purity of the PL with PC as predominant PL in the ethanol-soluble fraction, and less polar PL was dominant in the ethanolinsoluble fraction. The crude PL from PPF had a low HLB, and fractionation by ethanol produced a soluble fraction with a higher HLB value, but a lower value was found in the insoluble fraction. Further fractionation by acetone did not produce significantly different HLB values. It was found that crude PL from PPF was not suitable to stabilise $\mathrm{o} / \mathrm{w}$ emulsions. Ethanol and acetone fractionation resulted in better emulsifying properties and emulsion stability. Ethanol soluble fraction from PPF PL was a good candidate as an emulsifier for $\mathrm{o} / \mathrm{w}$ food emulsion systems.

Meanwhile, crude PPF PL and ethanol insoluble fraction were better for the w/o system. Fractionation by ethanol was sufficient to stabilise $\mathrm{o} / \mathrm{w}$ emulsion system. Crude PL from PPF and its fractions showed antioxidant activity in the bulk oil system and exhibited synergism with $\alpha$-tocopherol. Antioxidant activity was affected by PL composition in that the high level of PC had a significant contribution at preventing lipid oxidation in a single antioxidant system. PPF PL was better to use as an antioxidant in binary rather than single systems. Fractionation by ethanol followed by acetone was required to have better antioxidant activity.

\section{ACKNOWLEDGEMENT}

The authors would like to thank the Ministry of Research and Technology, the Republic of Indonesia for funding this research through Insentif Riset Dasar.

\section{REFERENCES}

Ahmad, M U and Xu, X (2015). Polar Lipids: Biology, Chemistry and Technology. AOCS Press, Urbana. 568 pp.

Alam, S; Algahtani, M S; Ahmad, M Z and Ahmad, $\mathrm{J}$ (2020). Investigation utilizing the HLB concept for the development of moisturizing cream and lotion: In-vitro characterization and stability evaluation. Cosmetics, 7(2): 1-12. DOI: $10.3390 /$ cosmetics7020043.

AOAC (1980). Analytical Chemistry. Official Methods of Analysis. $16^{\text {th }}$ edition. Association of Official Analytical Chemist (AOAC). Washington DC, USA.
AOAC (1997). Official Method 920.160. Saponification Number of Oils and Fats. Official Methods of Analysis. Rockville, USA.

Arnold, G; Schade, E; Schneider, Y; Friedrichs, J; Babick, F; Werner, C and Rohm, H (2014). Influence of individual phospholipids on the physical properties of oil-based suspensions. J. Amer. Oil Chem. Soc., 91(1): 71-77. DOI: 10.1007/ s11746-013-2361-6.

Belayneh, H D; Wehling, R L; Cahoon, E and Ciftci, O N (2018). Lipid composition and emulsifying properties of Camelina sativa seed lecithin. Food Chem., 242(7): 139-146. DOI: 10.1016/j. foodchem.2017.08.082.

Bernardo, J; Videira, R A; Valentão, P; Veiga, F and Andrade, P B (2019). Extraction of phospholipidrich fractions from egg yolk and development of liposomes entrapping a dietary polyphenol with neuroactive potential. Food Chem. Toxicol., 133(8): 110749. DOI: 10.1016/j.fct.2019.110749.

Cabezas, D M; Diehl, B W K and Tomás, M C (2009). Sunflower lecithin: Application of a fractionation process with absolute ethanol. J. Amer. Oil Chem. Soc., 86(2): 189-196. DOI: $10.1007 /$ s11746-0081336-5.

Cabezas, D M; Madoery, R; Diehl, B W K and Tomás, M C (2012). Emulsifying properties of different modified sunflower lecithins. J. Amer. Oil Chem. Soc., 89(2): 355-361. DOI: 10.1007/s11746-011-1915-8.

Cardenia, V; Waraho, T; Rodriguez-Estrada, M T; Julian McClements, D and Decker, E A (2011). Antioxidant and prooxidant activity behavior of phospholipids in stripped soybean oil-in-water emulsions. J. Amer. Oil Chem. Soc., 88(9): 1409-1416. DOI: 10.1007/s11746-011-1807-y.

Charanyaa, S; Belur, P D and Regupathi, I (2019). Effect of intrinsic and extrinsic factors on the storage stability of sardine oil. Curr. Res. Nutr. Food Sci., 7(3): 749-760. DOI: 10.12944/CRNFSJ.7.3.14.

Chen, J; Zhang, X; Xue, S and Xu, X (2020). Effects of ultrasound frequency mode on myofibrillar protein structure and emulsifying properties. Int. J. Biol. Macromol., 163: 1768-1779. DOI: 10.1016/j. ijbiomac.2020.09.114.

Choe, J and Choe, E (2016). Effect of soy-derived phospholipid on the autoxidation of canola oil in a water/oil emulsion. J. Amer. Oil Chem. Soc., 93(8): 1085-1094. DOI: 10.1007/s11746-016-2855-0.

Choo, Y M; Bong, S C; Ma, A N and Chuah, C H (2004). Phospholipids from palm-pressed fiber. 
J. Amer. Oil Chem. Soc., 81(5): 471-475. DOI: 10.1007/ s11746-004-0925-4.

Chua, S C; Tan, C P; Mirhosseini, H; Lai, O M; Long, K and Baharin, B S (2009). Optimization of ultrasound extraction condition of phospholipids from palm-pressed fiber. J. Food Eng., 92(4): 403-409. DOI:10.1016/j.jfoodeng.2008.12.013.

Cui, L and Decker, E A (2016). Phospholipids in foods: Prooxidants or antioxidants? J. Sci. Food Agric., 96(1): 18-31. DOI: 10.1002/ jsfa.7320.

Cui, L; Kittipongpittaya, K; McClements, D J and Decker, E A (2014). Impact of phosphoethanolamine reverse micelles on lipid oxidation in bulk oils. J. Amer. Oil Chem. Soc., 91(11): 1931-1937. DOI: 10.1007/ s11746-014-2544-9.

Doert, M; Jaworska, K; Moersel, J T and Kroh, L W (2012). Synergistic effect of lecithins for tocopherols: Lecithin-based regeneration of $\alpha$-tocopherol. Eur. Food Res. Technol., 235(5): 915-928. DOI: 10.1007/ s00217-012-1815-7.

Doert, M; Krüger, S; Morlock, G E and Kroh, L W (2017). Synergistic effect of lecithins for tocopherols: Formation and antioxidant effect of the phosphatidylethanolamine-l-ascorbic acid condensate. Eur. Food Res. Technol., 243(4): 583-596. DOI: $10.1007 /$ s00217-016-2768-z.

Estiasih, T; Ahmadi, K; Ginting, E and Priyanto, A D (2013). Modification of soy crude lecithin by partial enzymatic hydrolysis using phosholipase A1. Int. Food Res. J., 20(2): 843-849.

Feng, J; Chen, Q; Wu, X; Jafari, S M and McClements, D J (2018). Formulation of oil-in-water emulsions for pesticide applications: impact of surfactant type and concentration on physical stability. Environ. Sci. Pollut. Res., 25(22): 21742-21751. DOI: 10.1007/ s11356-018-2183-z.

Garba, U; Singanusong, R; Jiamyangyuen, $\mathrm{S}$ and Thongsook, T (2020). Extracting lecithin from water degumming by-products of rice bran oil and its physicochemical, antioxidant and emulsifying properties. Food Biosci., 38(9): 100745. DOI: 10.1016/j.fbio.2020.100745.

Guiotto, E N; Cabezas, D M; Diehl, B W K and Tomás, M C (2013). Characterization and emulsifying properties of different sunflower phosphatidylcholine enriched fractions. Eur. J. Lipid Sci. Technol., 115(8): 865-873. DOI: 10.1002/ ejlt.201200394.

Hills, G L and Thiel, C C (1946). The ferric thiocyanate method of estimating peroxide in the fat of butter, milk and dried milk. J. Dairy Res., 14(340-353). DOI: 10.1017 / s0022029900004799.

Lau, H L N; Choo, Y M; Ma, A N and Chuah, C H (2006). Quality of residual oil from palm-pressed mesocarp fiber (Elaeis guineensis) using supercritical $\mathrm{CO} 2$ with and without ethanol. J. Amer. Oil Chem. Soc., 83(10): 893-898. DOI: 10.1007 / s11746-006-50439.

Li, J; He, Y; Anankanbil, S and Guo, Z (2019). Phospholipid-based surfactants. in Biobased Surfactants: Synthesis, Properties and Applications (Hayes, D G; Solaiman, D K Y and Ashby, R D eds.). Elsevier Inc, New York. p. 243-286.

Malaplate, C; Poerio, A; Huguet, M; Soligot, C; Passeri, E; Kahn, C J F; Linder, M; Arab-Tehrany, $\mathrm{E}$ and Yen, F T (2019). Neurotrophic effect of fishlecithin based nanoliposomes on cortical neurons. Mar. Drugs, 17(7): 1-13. DOI: 10.3390 /md17070406.

McClements, D J and Gumus, C E (2016). Natural emulsifiers - Biosurfactants, phospholipids, biopolymers and colloidal particles: Molecular and physicochemical basis of functional performance. Adv. Colloid Interface Sci., 234: 3-26. DOI: 10.1016/j. cis.2016.03.002.

Nagai, K (2012). Bovine milk phospholipid fraction protects Neuro2a cells from endoplasmic reticulum stress via PKC activation and autophagy. J. Biosci. Bioeng., 114(4): 466-471. DOI: 10.1016/j. jbiosc.2012.05.009.

Nzai, J M and Proctor, A (1998). Phospholipids determination in vegetable oil by thin-layer chromatography and imaging densitometry. Food Chem., 63(4): 571-576. DOI: 10.1016/s03088146(98)00043-0.

Ooi, J; Promentilla, M A B; Tan, R R; Ng, D K S and Chemmangattuvalappil, N G (2019). Alternative solvent design for oil extraction from palm pressed fibre via computer-aided molecular design. Technologies for the Oil Palm Industry (Foo, C Y D and Tun Abdul Aziz eds.). Green Spinger, Singapore. p. 33-55.

Palacios, L E and Wang, T (2005). Extraction of eggyolk lecithin. J. Amer. Oil Chem. Soc., 11: 565-569. DOI: 10.1007/s11746-005-1110-5.

Pearce, K N and Kinsella, J E (1978). Emulsifying properties of proteins: Evaluation of a turbidimetric technique. J. Agric. Food Chem., 26(3): 716-723.

Robert, C; Couëdelo, L; Vaysse, C and Michalski, 
M C (2020). Vegetable lecithins: A review of their compositional diversity, impact on lipid metabolism and potential in cardiometabolic disease prevention. Biochimie, 169: 121-132. DOI: 10.1016/j. biochi.2019.11.017.

Silva, M and Lidon, F (2016). An overview on applications and side effects of antioxidant food additives. Emir. J. Food Agric., 28(12): 823. DOI: 10.9755/ejfa.2016-07-806.

Sun, X; Zhang, L; Tian, S; Yang, K and Xie, J (2020). Phospholipid composition and emulsifying properties of rice bran lecithin from enzymatic degumming. LWT - Food Sci. Technol., 117(8): 108588. DOI: 10.1016/j.lwt.2019.108588.

Topuz, O K; Aygün, T; Alp, A C; Yatmaz, H A; Torun, M and Yerlikaya, P (2021). Characterization and emulsifying properties of aquatic lecithins isolated from processing discard of rainbow trout fish and its eggs. Food Chem., 339(9): 128103. DOI: 10.1016/j. foodchem.2020.12810.

Warren, P B; Shin, S and Stone, H A (2019). Diffusiophoresis in ionic surfactants: Effect of micelle formation. Soft Matter, 15(2): 278-288. DOI: 10.1039 / c8sm01472h.

$\mathrm{Wu}, \mathrm{D}$; Lu, J; Zhong, S; Schwarz, P; Chen, B and Rao, J (2019). Effect of chitosan coatings on physical stability, antifungal and mycotoxin inhibitory activities of lecithin stabilized cinnamon oil-inwater emulsions. LWT - Food Sci. Technol., 106(1): 98104. DOI: $10.1016 /$ j.lwt.2019.02.029.

Xie, M and Dunford, N T (2019). Fractionating of canola lecithin from acid degumming and its effect. Food Chem., 300(6): 125217. DOI: 10.1016/j. foodchem.2019.125217.

Yunoki, K; Kukino, O; Nadachi, Y; Fujino, $\mathrm{T}$ and Ohnishi, M (2008). Separation and determination of functional complex lipids from chicken skin. J. Amer. Oil Chem. Soc., 85(5): 427-433. DOI: 10.1007/s11746008-1228-8.

Zembyla, M; Murray, B S and Sarkar, A (2020). Water-in-oil emulsions stabilized by surfactants, biopolymers and/or particles: A review. Trends Food Sci. Technol., 104(7): 49-59. DOI: 10.1016/j. tifs.2020.07.028. 